\title{
THE IDEAL CENTER OF THE DUAL OF A BANACH LATTICE
}

\author{
MEHMET ORHON \\ Dedicated to Professor W.A.J. Luxemburg on the occasion of his 80th birthday

\begin{abstract}
Let $E$ be a Banach lattice. Its ideal center $Z(E)$ is embedded naturally in the ideal center $Z\left(E^{\prime}\right)$ of its dual. The embedding may be extended to a contractive algebra and lattice homomorphism of $Z(E)^{\prime \prime}$ into $Z\left(E^{\prime}\right)$. We show that the extension is onto $Z\left(E^{\prime}\right)$ if and only if $E$ has a topologically full center. (That is, for each $x \in E$, the closure of $Z(E) x$ is the closed ideal generated by $x$.) The result can be generalized to the ideal center of the order dual of an Archimedean Riesz space and in a modified form to the orthomorphisms on the order dual of an Archimedean Riesz space.
\end{abstract}

\section{INTRODUCTION}

Let $E$ be a Banach lattice and let $Z(E)$ denote its (ideal) center. In general $Z(E)$ is a subalgebra and a sublattice of $Z\left(E^{\prime}\right)$, the center of the dual of E. The embedding may be extended to a contractive algebra and lattice homomorphism of $Z(E)^{\prime \prime}$ into $Z\left(E^{\prime}\right)$. In this paper we will show that the extension is onto $Z\left(E^{\prime}\right)$ if and only if $E$ has a topologically full center. (That is, for each $x \in E$, the closure of $Z(E) x$ is the closed ideal generated by $x$.) In this case $Z\left(E^{\prime}\right)$ is isomorphic, both as an algebra and as a vector lattice, to a band in $Z(E)^{\prime \prime}$.

Let $K$ be a compact Hausdorff space and let $C(K)$ denote the Banach algebra of continuous functions on $K$ with the sup norm. Also let $L(E)$ denote the bounded linear operators on $E$ and let $m: C(K) \rightarrow L(E)$ be a bounded unital algebra homomorphism. If each closed $C(K)$-invariant subspace of $E$ is an ideal, we will call $m$ an ideal generating representation of $C(K)$ on $E$. The main result of this paper asserts that an ideal generating representation of $C(K)$ uniquely determines the center of both $E$ and $E^{\prime}$. Moreover, up to lattice isometry, an ideal generating representation determines the order structure of $E$ as well.

The center of a Banach lattice has been of considerable importance in the study of operators on Banach lattices, especially in proving dominance theorems. Several definitions have been given in the literature of conditions expressing that the center is large in some sense. Important examples include Meyer's topological richness [12, Hart's transitivity [8] and Wickstead's topological fullness [17. For our purposes, topological fullness is the most useful of these concepts.

The result stated in the first paragraph can be generalized to the ideal center of the order dual of a Riesz space and, in a modified form, to the orthomorphisms

Date: January 31, 2010.

2000 Mathematics Subject Classification. Primary 47B38, 47B60, 46B42; Secondary 46H25, $47 \mathrm{~L} 10$

Key words and phrases. Banach lattice, ideal center, topologically full, order ideal, Banach $C(K)$-module. 
on the order dual of a Riesz space when the order dual separates the points of the Riesz space.

All standard terminology and standard results about vector lattices that we use may be found in at least one of [2, 11], 15] or 20]. Except in Remark 5, we do not make a distinction between the real and the complex spaces. Our results hold in either case.

\section{IDEAL GENERATING REPRESENTATIONS}

Suppose a bounded unital homomorphism $m: C(K) \rightarrow L(E)$ is given. Then we consider its Arens extension $m^{*}: C(K)^{\prime \prime} \rightarrow L\left(E^{\prime}\right)$ as follows. Associated with the homomorphism $m$, we define three bilinear maps:

$$
\begin{aligned}
& C(K) \times E \rightarrow E::(a, x) \rightarrow a x: a x=m(a)(x) \\
& E \times E^{\prime} \rightarrow C(K)^{\prime}::\left(x, x^{\prime}\right) \rightarrow \mu_{x x^{\prime}}: \mu_{x x^{\prime}}(a)=x^{\prime}(a x) \\
& E^{\prime} \times C(K)^{\prime \prime} \rightarrow E^{\prime}::\left(x^{\prime}, a\right) \rightarrow a x^{\prime}: a x^{\prime}(x)=a\left(\mu_{x x^{\prime}}\right) .
\end{aligned}
$$

We recall that $C(K)^{\prime \prime}$ is isomorphic to $C(S)$ with $S$ hyperstonian. Then it is routine to show that

$$
m^{*}: C(K)^{\prime \prime} \rightarrow L\left(E^{\prime}\right): m^{*}(a)\left(x^{\prime}\right)=a x^{\prime}
$$

is a bounded unital algebra homomorphism that is $\left(w^{*}, w^{*}\right.$-operator $)$-continuous. Also for each $a \epsilon C(K), m^{*}(a)$ is the adjoint in $L\left(E^{\prime}\right)$ of the operator $m(a)$ in $L(E)$. For information on Arens extensions see [3].

Recall that $T \epsilon L(E)$ is in the center $Z(E)$ of a Banach lattice $E$ if there is an $M>0$ such that for each $x \epsilon E_{+}$, we have $|T(x)| \leq M x$. (Evidently one can take $M=\|T\|$.) In what follows we will use a number of known results about the center of a Banach lattice. We mention them here briefly. An operator $T$ on $E$ is in $Z(E)$ if and only if $T$ leaves each closed ideal of $E$ invariant [16. If $E$ is Dedekind complete then less is required. In this case, $T$ is in $Z(E)$ if and only if $T$ leaves each band of $E$ invariant. This means that when $E$ is Dedekind complete then an operator $T$ on $E$ is in $Z(E)$ if and only if $T$ commutes with each band projection on $E$. Each band projection is in $Z(E)$. Consequently, one has the well known result that when $E$ is Dedekind complete then an operator $T$ is in $Z(E)$ if and only if $T$ commutes with all the operators in $Z(E)$. Since the dual $E^{\prime}$ of a Banach lattice $E$ is Dedekind complete, the above statements apply in particular to $Z\left(E^{\prime}\right)$. Finally, the definition of membership in the ideal center and the order structure of the dual imply that an operator $T$ on $E$ is in $Z(E)$ if and only if its adjoint $T^{\prime}$ is in $Z\left(E^{\prime}\right)$.

Let $A$ be a subset of $L(E)$, we denote by $w-\operatorname{cl}(A)$ the weak-operator closure of $A$ in $L(E)$.

Theorem 1. Let $E$ be a Banach lattice, $K$ be a compact Hausdorff space and $m: C(K) \rightarrow L(E)$ be a bounded unital algebra homomorphism. Consider the following statements:

(1) Each closed $C(K)$-invariant subspace of $E$ is an ideal.

(2) $Z\left(E^{\prime}\right)=m^{*}\left(C(K)^{\prime \prime}\right)$.

(3) $Z(E)=w-\operatorname{cl}(m(C(K)))$.

Then $(1) \Leftrightarrow(2) \Rightarrow(3)$. Also, in this case, $m$ is a positive contractive homomorphism. 
Proof. Assume (1) holds. Then, by duality, each $w^{*}$-closed $C(K)$-invariant subspace of $E^{\prime}$ is a band. Therefore any operator in $Z\left(E^{\prime}\right)$ leaves such a subspace invariant. But, since $C(K)$ is $w^{*}$-dense in $C(K)^{\prime \prime}$, the continuity properties of $m^{*}$ imply that a $w^{*}$-closed $C(K)$-invariant subspace of $E^{\prime}$ is in fact $C(K)^{\prime \prime}$-invariant. We can summarize the discussion with the statement that each $w^{*}$-closed $C(K)^{\prime \prime}$ invariant subspace of $E^{\prime}$ is left invariant by each operator in $Z\left(E^{\prime}\right)$. Then by a deep result due to Arenson ([1, 9.12, page 63), $Z\left(E^{\prime}\right)$ is contained in $m^{*}\left(C(K)^{\prime \prime}\right)$. (See Remark 1 below for a more detailed explanation.) Since $Z\left(E^{\prime}\right)$ is contained in $m^{*}\left(C(K)^{\prime \prime}\right)$ and since $m^{*}\left(C(K)^{\prime \prime}\right)$ is a commutative subalgebra of $L\left(E^{\prime}\right)$, each operator in $m^{*}\left(C(K)^{\prime \prime}\right)$ commutes with all the operators in $Z\left(E^{\prime}\right)$. It follows from the discussion preceeding Theorem 1 that $m^{*}\left(C(K)^{\prime \prime}\right)$ is, in turn, a subset of $Z\left(E^{\prime}\right)$. That is, $(1) \Rightarrow(2)$.

Conversely, assume (2) holds. Suppose $F$ is a closed $C(K)$-invariant subspace of $E$. Consider its annihilator $F^{\circ}$ in $E^{\prime} . F^{\circ}$ is a $w^{*}$-closed $C(K)$-invariant subspace of $E^{\prime}$. Then, as in the proof of $(1) \Rightarrow(2), F^{\circ}$ is a $w^{*}$-closed $C(K)^{\prime \prime}$-invariant subspace of $E^{\prime}$ by the continuity properties of $m^{*}$. Now, by (2), we have that $F^{\circ}$ is a $w^{*}$-closed $Z\left(E^{\prime}\right)$-invariant subspace of $E^{\prime}$. Since $E^{\prime}$ is Dedekind complete, a $Z\left(E^{\prime}\right)$-invariant subspace of $E^{\prime}$ is an ideal. Therefore $F^{\circ}$ is an ideal in $E^{\prime}$. It follows from duality that $F$ is an ideal in $E$. That is, $(2) \Rightarrow(1)$.

The kernel of $m^{*}$ is a $w^{*}$-closed band in $C(K)^{\prime \prime}$. Therefore, when (2) holds, the idempotents in $C(K)^{\prime \prime}$ are mapped onto the band projections in $Z\left(E^{\prime}\right)$. Since these generate $C(K)^{\prime \prime}$ and $Z\left(E^{\prime}\right)$ respectively, (2) implies that $m^{*}$ is contractive and positive. In fact if $(1-e)$ denotes the band projection in $C(K)^{\prime \prime}$ onto the kernel of $m^{*}$ then $Z\left(E^{\prime}\right)$ is isometrically isomorphic to $e C(K)^{\prime \prime}$ both as an algebra and as a Banach lattice. Also, to see $(2) \Rightarrow(3)$, note that $(2)$ and the continuity properties of $m^{*}$ imply that $Z(E)=w-c l(m(C(K)))$.

We recall that the center of a Banach lattice $E$ is isomorphic to $C(K)$ for some compact Hausdorff space $K$ both as an algebra and a vector lattice. Moreover the center is closed in $L(E)$ with respect to the weak-operator topology . Also recall that a Banach lattice $E$ is said to have a topologically full center if, for each $x \epsilon E_{+}$, the closure of $Z(E) x$ is an ideal in $E$ [17]. This leads to:

Corollary 2. Let $E$ be a Banach lattice and let $i: Z(E) \rightarrow L(E)$ denote the natural embedding of $Z(E)$ into $L(E)$. Then $E$ has a topologically full center if and only if $i^{*}\left(Z(E)^{\prime \prime}\right)=Z\left(E^{\prime}\right)$. When that holds we may identify $Z\left(E^{\prime}\right)$ with a band in $Z(E)^{\prime \prime}$ both as an algebra and as a Banach lattice.

Banach lattices with a topological order unit (that is, with a quasi-interior element) and $\sigma$-Dedekind complete Banach lattices are examples of Banach lattices with topologically full center. Not all Banach lattices have topologically full center. In particular a Banach lattice with trivial center does not have a topologically full center unless the vector lattice is one dimensional [6, 18. This shows that in general the inclusion of $i^{*}\left(Z(E)^{\prime \prime}\right)$ in $Z\left(E^{\prime}\right)$ may be strict and that one can not expect $(3) \Rightarrow(2)$ to hold in Theorem 1.

It is well known that if a Banach lattice is $\sigma$-Dedekind complete then its center is maximal abelian in the algebra of bounded operators on the Banach lattice, e.g., 19. It follows from this fact and Corollary 2 that if the center of a Banach lattice is topologically full then the center is maximal abelian in the algebra of bounded 
operators on the Banach lattice. Also, recently Wickstead [19] has given a direct proof of the result that does not require duality arguments.

Corollary 3. Let $E$ be a Banach lattice with topologically full center and let $T$ be an operator on $E$ that commutes with $Z(E)$. Then $T$ is in $Z(E)$.

Proof. Consider $T^{\prime}$, the adjoint of $T$ in $L\left(E^{\prime}\right)$. Then the continuity properties of $i^{*}$ and of $T^{\prime}$ imply that $T^{\prime}$ commutes with $i^{*}\left(Z(E)^{\prime \prime}\right)=Z\left(E^{\prime}\right)$. Since $E^{\prime}$ is Dedekind complete, $T^{\prime} \in Z\left(E^{\prime}\right)$. Therefore $T \in Z(E)$ by the discussion preceeding Theorem 1.

Next, we show that an ideal generating representation of $C(K)$ on a Banach lattice $E$ determines the order structure of $E$ up to lattice isomorphism. The actual result is true more generally. In the proof below $C_{\infty}(K)=\{f+i g$ : with $f, g \in$ $\left.C_{\infty}(K)_{r}\right\}$ where $C_{\infty}(K)_{r}$ denotes the set of all extended continuous functions on $K$ into $[-\infty, \infty]$, the two point compactification of the real numbers. An extended continuous function on $K$ is a continuous function into $[-\infty, \infty]$ that is finite except possibly on a nowhere dense subset of $K([15$, , 19, [13]).

Theorem 4. Let $E$ be a Banach lattice and let $W$ be a cone in $E$ with the properties:

(1) $E$ is a Banach lattice with the cone $W$ and with its original norm,

(2) $E$ has the same closed ideals with respect to the cones $E_{+}$and $W$.

Then there is $T \epsilon Z(E)$ with $|T|=1$ such that $T(x)=/ x / W$ for each $x \epsilon E_{+}$. (Here $/ . / W$ denotes absolute value with respect to the lattice structure given by the cone $W$.) Conversely, if $T \epsilon Z(E)$ with $|T|=1$, then $W=T\left(E_{+}\right)$gives a cone in $E$ such that (1) and (2) hold.

Proof. Note that, since $E_{+}$and $W$ give the same closed ideals in $E$, the ideal center with respect to either lattice structure consists of the same operators. In fact the order structure of the ideal center is the same in both cases. This latter statement follows from Theorem 1 if $E$ has a topological order unit. To see it for arbitrary $E$, apply the case of a Banach lattice with topological order unit to the closure of each principal ideal in $E$ with $Z(E)$ restricted to each such ideal. Therefore $Z(E)=C(K)$ for some compact Hausdorff space $K$ and $Z(E)_{+}=C(K)_{+}$in both lattice structures. Denote by $I(x)$ the closed ideal generated by $x \epsilon E$ in either lattice structure.

Suppose initially that $E$ has a topological order unit $u$ with respect to $E_{+}$. Then $/ u / W$ is a topological order unit with respect to $W$. To see this observe that $I(u)=I(/ u / W)$. Represent the Banach lattice with the cone $W$ as a sublattice of $C_{\infty}(K)$ such that $/ u / W$ is represented by 1 and $C(K)$ with its lattice structure corresponds to the ideal generated by $/ u /{ }_{W}$ ([15], III.4.5). Let $f \epsilon C(K)$ be the function that represents $u$. Then $|f|=1$. Let $T \epsilon Z(E)$ be the operator that corresponds to $\bar{f} \epsilon C(K)$. It follows from the functional representation that $T(u)=/ u / W$. For each $a \epsilon C(K)_{+}$, one has $T(a u)=a T(u)=a / u / W=/ a u / W$. Then it follows that $T(x)=/ x / W$ for all $x \epsilon E_{+}$when $E$ has a topological order unit. Now repeat the above argument for any $a \epsilon C(K)$, to show that $T(|x|)=/ T(x) /{ }_{W}(=/ x / W)$ for all $x \epsilon E$. That is, $T$ is indeed a lattice homomorphism between the two lattice structures.

In the general case let, $E=\bigcup_{x \epsilon E_{+}} I(x)$ where the family $\left\{I(x): x \epsilon E_{+}\right\}$is directed upwards by inclusion in the order of $E_{+}$. Since $I(x)=I(/ x / W)$ for each $x \in E_{+}$, by the case when there is a topological order unit, there is $T_{x} \in Z(I(x))$ 
such that $T_{x}(z)=/ z /{ }_{W}$ for all $z \epsilon I(x)_{+}$. Note that $0 \leq y \leq x$ for some $x, y \in E_{+}$ implies that $T_{x}$ is an extension of $T_{y}$. Therefore, without ambiguity, we define an operator $T$ of norm 1 on $E$ by $T(z)=T_{x}(z)$ whenever $z \epsilon I(x)$ for some $x \epsilon E_{+}$. Since $T$ preserves closed ideals, $T \in Z(E)$. Also it is clear from the definition of $T$ that $|T|=1$. This completes the first part of the proof.

The converse on the other hand is a routine exercise. Let $/ x / W=T(|x|)$ for each $x \epsilon E$ and check that (1) and (2) are satisfied.

Remark 1. For the purpose of consistency and clarity we will restate Arenson's Theorem ([1, 9.12) in our notation and terminology: Let $E$ be a Banach space and let $m: C(K) \rightarrow L(E)$ be a bounded unital algebra homomorphism. Then an operator $T$ on $E^{\prime}$ is in $m^{*}\left(C(K)^{\prime \prime}\right)$ if and only if $T$ leaves invariant each $w^{*}$-closed $C(K)^{\prime \prime}$-invariant subspace of $E^{\prime}$. In order to see that our restatement is faithful, note that the continuity properties of $m^{*}$ and basic duality theory show that $Z\left(X^{*}\right)$ involved in the statement of Arenson's Theorem for Banach $C(K)$-modules is equal to $m^{*}\left(C(K)^{\prime \prime}\right)$ in our setting $(\mathbb{1}, 7.71$, p. 46). The proof of the theorem uses several other results in [1] that are remarkable in their own right. In particular the proof uses an analogue of the Factorization Theorem of Lozanovsky on Banach function spaces $[10,5$ that is proved in 1 for Banach $C(K)$-modules. For an earlier version of Arenson's Theorem in the resricted setting of Bade's Theorem see ([14], Theorem 2), for a weaker version see ([7, Lemma 5). This latter result states (in the same circumstances as Arenson's Theorem): If an operator $T$ on $E^{\prime}$ leaves invariant each $w^{*}$-closed $C(K)^{\prime \prime}$-invariant subspace of $E^{\prime}$ and commutes with $m^{*}\left(C(K)^{\prime \prime}\right)$ then $T$ is in $m^{*}\left(C(K)^{\prime \prime}\right)$. Its proof is considerably simpler than that of Arenson's Theorem and it may be used to provide an alternative proof of Theorem 1 .

In the next remark we will outline the above mentioned alternative proof of Theorem 1.

Remark 2. To give a different proof of Theorem 1, we only need to prove (1) implies (2) without using Arenson's Theorem. Assume (1) in the statement of Theorem 1. Let $a \in C(K)$, then the kernel of $m^{*}(a)$ is a $w^{*}$-closed $C(K)^{\prime \prime}$-invariant subspace of $E^{\prime}$ and is a band. Therefore $Z\left(E^{\prime}\right)$ leaves $\operatorname{Ker}\left(m^{*}(a)\right)$ invariant for all $a \in C(K)$. That is, for all $T \in Z\left(E^{\prime}\right), a \in C(K)$ and $x^{\prime} \in E^{\prime}$,

$$
m^{*}(a)\left(x^{\prime}\right)=0 \text { implies } m^{*}(a)\left(T\left(x^{\prime}\right)\right)=0 .
$$

Then by a result of Evans 4, each operator in $Z\left(E^{\prime}\right)$ commutes with all the operators in $m^{*}(C(K))$. (See also [1, 9.5.) Since $E^{\prime}$ is Dedekind complete, we have $m^{*}(C(K)) \subset Z\left(E^{\prime}\right)$. By the Hahn-Banach Theorem, the set of non-negative elements of the unit ball of $C(K)$ are $w^{*}$-dense in the set of non-negative elements of the unit ball of $C(K)^{\prime \prime}$. Now, the continuity properties of the homomorphism $m^{*}$ and the definition of membership in the ideal center imply that $m^{*}\left(C(K)^{\prime \prime}\right) \subset$ $Z\left(E^{\prime}\right)$. This means that $Z\left(E^{\prime}\right)$ leaves invariant each $w^{*}$-closed $C(K)^{\prime \prime}$-invariant subspace of $E^{\prime}$ and commutes with $m^{*}\left(C(K)^{\prime \prime}\right)$. Then Lemma 5 [7] implies that $Z\left(E^{\prime}\right) \subset m^{*}\left(C(K)^{\prime \prime}\right)$. This completes the proof.

Remark 3. It is natural to ask if the converse of Corollary 3 is true. A.W. Wickstead has given an example of a Banach lattice that does not have a topologically full center, but whose center is maximal abelian [19. Wickstead's paper contains several 
interesting problems related to Banach lattices with maximal abelian center or with topologically full center.

Remark 4. When $E$ is a Banach lattice with topological order unit a much stronger result than Corollary 3 is proved in $\left([1,9.11,(1))\right.$ : Let $T$ be an operator on $E^{\prime}$ that commutes with $i^{*}(Z(E))$, then $T$ is in $Z\left(E^{\prime}\right)$. It is further stated in 1 that the result remains true if $E$ is a Banach lattice with topologically full center. However this latter statement is still an open problem. For a more general version of this problem, the interested reader should see $([7,3 .$, p. 357). It is worth noting that the other two questions raised in $(\mathbf{7}, 3$.) have been answered affirmatively by Arenson's Theorem.

Remark 5. Let $E$ be a Riesz space with point separating order dual $E^{\sim}$. We will say that $E$ has a topologically full center, if for each $x \epsilon E_{+}$, the weak closure of $Z(E) x$ is an ideal. Let $L_{b}(E)$ denote the order bounded operators on $E$. Let $i: Z(E) \rightarrow$ $L_{b}(E)$ be the natural embedding and let $i^{\sim}: Z(E)^{\prime \prime} \rightarrow L_{b}\left(E^{\sim}\right)$ be its Arens extension. Then $E$ has topologically full center if and only if $i^{\sim}\left(Z(E)^{\prime \prime}\right)=Z\left(E^{\sim}\right)$. Let $\operatorname{Orth}(E)$ denote the orthomorphisms on $E$. (That is, the band preserving operators in $L_{b}(E)$.) Let $E_{n}^{\sim}$ denote the order continuous linear functionals on $E$. Let $\gamma: \operatorname{Orth}(E) \rightarrow L_{b}(E)$ be the natural embedding and let its Arens extension be denoted by $\gamma^{\sim}:\left(\operatorname{Orth}(E)^{\sim}\right)_{n}^{\sim} \rightarrow L_{b}\left(E^{\sim}\right)$. Then $E$ has topologically full center if and only if $\gamma^{\sim}\left(\left(\operatorname{Orth}(E)^{\sim}\right)_{n}^{\sim}\right)$ is an ideal in $\operatorname{Orth}\left(E^{\sim}\right)$. In the case of orthomorphisms we do not need the bidual since $\left(\operatorname{Orth}(E)^{\sim}\right)_{n}^{\sim}$ is the bidual of $\operatorname{Orth}(E)$ [9]. The motivation for the results stated in this remark is the work of Huijsmans and de Pagter 9 on the Arens product on the bidual of an f-algebra. The above statements are proved using the results developed in $[9$ and elementary duality theory.

I wish to thank A.W. Wickstead for his valuable comments and the referee for suggesting the inclusion of the alternative proof of Theorem 1 and the remarks on the open questions.

\section{REFERENCES}

[1] Y. A. Abramovich, E. L. Arenson and A. K. Kitover, Banach $C(K)$-modules and operators preserving disjointness, Pitman Research Notes, 277, Wiley, New York, 1992.

[2] C. D. Aliparantis and O. Burkinshaw, Positive operators, Academic Press, New York, 1985.

[3] R. Arens, The adjoint of a bilinear operation, Proc. Amer. Math. Soc. 2(1951), 839-848.

[4] R. Evans, Embedding $C(K)$ in B(X), Math. Scand. 48(1981), 119-136.

[5] T. A. Gillespie, Factorization in Banach function spaces, Nederl. Akad. Wetensch. Indag. Math. 43(1981), 287-300.

[6] A. Goullet de Rugy, La structure idéale des M-espaces, J. Math. Pures Appl. 51(1972), 331-337.

[7] D. Hadwin and M. Orhon, Reflexivity and approximate reflexivity for Boolean algebras of projections, J. Func. Anal. 87(1989), 348-358.

[8] D. R. Hart, Disjointness preserving operators, Ph. D. Thesis., Pasadena: California Institute of Technology, 1983.

[9] C. B. Huijsmans and B. de Pagter, The order bidual of lattice ordered algebras, J. Func. Anal. 59(1984), 41-64

[10] G. Ya. Lozanovsky, On some Banach lattices, Sibirsk. Mat. Zh. 10(1969), 584-599.

[11] W. A. J. Luxemburg and A. C. Zaanen, Riesz spaces I, North Holland, Amsterdam, 1971.

[12] M. Meyer, Richesses du centre d'un espace vectoriel réticulé, Math. Ann. 236(1978), 147-169.

[13] H. Önder and M. Orhon, Transitive operator algebras on the $n$-fold direct sum of a cyclic Banach space, J. Operator Theory 22(1989), 99-107. 
[14] M. Orhon, Boolean algebras of commuting projections, Math. Z. 183(1983), 531-537.

[15] H. H. Schaefer, Banach lattices and positive operators, Springer, Berlin, 1974.

[16] A.W. Wickstead, The structure space of a Banach lattice, J. Math. Pures Appl. (9)56(1977), $33-45$.

[17] A. W. Wickstead, Extremal structure of cones of operators, Quart. J. Math. Oxford (2)32(1981), 239-253.

[18] A. W. Wickstead, Banach lattices with a trivial centre, Proc. Roy. Irish Acad., Sect. A 88(1988), 71-83.

[19] A. W. Wickstead, Banach lattices with topologically full centre, Vladikavkaz Mathematical Journal 11(2009), 50-60.

[20] A. C. Zaanen, Riesz spaces II, North Holland, Amsterdam, 1983.

Department of Mathematics \& Statistics, University of New Hampshire, Durham, NH 03824

E-mail address: mo@unh.edu 